\title{
LAPLACIAN DISTRIBUTION AND DOMINATION
}

\author{
DOMINGOS M. CARDOSO, DAVID P. JACOBS, AND VILMAR TREVISAN
}

\begin{abstract}
Let $m_{G}(I)$ denote the number of Laplacian eigenvalues of a graph $G$ in an interval $I$, and let $\gamma(G)$ denote its domination number. We extend the recent result $m_{G}[0,1) \leq \gamma(G)$, and show that isolate-free graphs also satisfy $\gamma(G) \leq m_{G}[2, n]$. In pursuit of better understanding Laplacian eigenvalue distribution, we find applications for these inequalities. We relate these spectral parameters with the approximability of $\gamma(G)$, showing that $\frac{\gamma(G)}{m_{G}[0,1)} \notin O(\log n)$. However, $\gamma(G) \leq m_{G}[2, n] \leq(c+1) \gamma(G)$ for $c$-cyclic graphs, $c \geq 1$. For trees $T, \gamma(T) \leq m_{T}[2, n]<2 \gamma(G)$.
\end{abstract}

Key words and phrases: graph, Laplacian eigenvalue, domination number. AMS subject classification: 05C50, 05C69.

\section{INTRODUCTION}

Let $G=(V, E)$ be an undirected graph with vertex set $V=\left\{v_{1}, \ldots, v_{n}\right\}$. For $v \in V$, its open neighborhood $N(v)$ denotes the set of vertices adjacent to $v$. The adjacency matrix of $G$ is the $n \times n$ matrix $A=\left[a_{i j}\right]$ for which $a_{i j}=1$ if $v_{i}$ and $v_{j}$ are adjacent, and $a_{i j}=0$ otherwise.

The Laplacian matrix of $G$ is defined as $L_{G}=D-A$, where $D=\left[d_{i j}\right]$ is the diagonal matrix in which $d_{i i}=\operatorname{deg}\left(v_{i}\right)$, the degree of $v_{i}$. The Laplacian spectrum of $G$ is the multi-set of eigenvalues of $L_{G}$, we number

$$
\mu_{1} \geq \mu_{2} \geq \ldots \geq \mu_{n}=0 .
$$

It is known that $\mu_{1} \leq n$. Unless indicated otherwise, all eigenvalues in this paper are Laplacian. We refer to [22, 23] for more background on the Laplacian spectra of graphs.

A set $S \subseteq V$ is dominating if every $v \in V-S$ is adjacent to some member in $S$. The domination number $\gamma(G)$ is the minimum size of a dominating set. Its decision problem is well-known to be NP-complete, and it is even hard to approximate.

Since 1996, several papers have been written relating the Laplacian spectrum of a graph $G$ with $\gamma(G)$. Often these results obtain a bound, involving $\gamma(G)$, for a specific eigenvalue such as $\mu_{1}$ or $\mu_{n-1}$. For example, it was shown that $\mu_{1}<n-\left\lceil\frac{\gamma(G)-2}{2}\right\rceil$ by Brand and Seifter [6] for $G$ connected and $\gamma(G) \geq 3$. This was recently improved in [26]. We refer to the introduction of [18] for a summary of these results.

Other spectral graph theory papers, including this one, are interested in distribution, that is, the number of Laplacian eigenvalues in an interval. For a real interval $I, m_{G}(I)$ denotes the number of Laplacian eigenvalues of $G$ in $I$. There exist several papers in the literature that relate Laplacian distribution to specific graph parameters, including $\gamma(G)$. For example, the paper by Zhou, Zhou and Du [27] shows that for trees $T$, $m_{T}[0,2) \leq n-\gamma(T)$.

The following spectral lower bound for $\gamma(G)$ was proved in [18]:

Domingos M. Cardoso was partially supported by the Portuguese Foundation for Science and Technology (FCT-Fundação para a Ciência e a Tecnologia), through the CIDMA - Center for Research and Development in Mathematics and Applications, within project UID/MAT/04106/2013.

David P. Jacobs and Vilmar Trevisan were supported by CNPq Grant 400122/2014-6, Brazil. 
Theorem 1. If $G$ is a graph, then $m_{G}[0,1) \leq \gamma(G)$.

In this paper we observe that for $G$ isolate-free one has

$$
\gamma(G) \leq m_{G}[2, n] .
$$

Since $m_{G}[0,2)+m_{G}[2, n]=n$, this inequality generalizes the result in [27] for trees.

Our paper seeks applications to the inequalities $m_{G}[0,1) \leq \gamma(G)$ and $\gamma(G) \leq m_{G}[2, n]$. We also seek insight into the ratios of these numbers. In the examples given in [18], the numbers $\gamma(G)$ and $m_{G}[0,1)$ were equal or differed by one. We will see that this does not happen in general.

The remainder of our paper is organized as follows. We finish this introduction by considering the sharpness of these inequalities. In the next section we recall the proof of Theorem 1 and modify it to obtain an inequality involving $m_{G}[2, n]$. In Section 3 we obtain several new results based on existing Nordhaus-Gaddum inequalities and Gallaitype theorems. One interesting new Nordhaus-Gaddum result is that for any graph $G$, $m_{G}[0,1)+m_{\bar{G}}[0,1) \leq n+1$ with equality if and only if $G=K_{n}$ or $G=\bar{K}_{n}$. Another interesting result is that a graph must have fewer than $\sqrt{n}$ Laplacian eigenvalues in at least one of the intervals $[0,1)$ or $(n-1, n]$. In Section 4 , using results from the approximation literature, we explain why we can't expect the quantities $m_{G}[0,1)$ or $m_{G}[2, n]$ to be close to $\gamma(G)$. Using some results on Vizing's conjecture, we show that $\frac{\gamma(G)}{m_{G}[0,1)} \notin O(\log n)$. For trees, $\gamma(T) \leq m_{T}[2, n]<2 \gamma(T)$. For $c$-cyclic graphs $G, c \geq 1$, $m_{G}[2, n] \leq(c+1) \gamma(G)$. These results seem interesting in light of the domination number's general inapproximability. In Section 5 we observe that many results also hold for the signless Laplacian spectrum.

Tightness. We briefly discuss whether $\gamma(G)$ is the natural graph parameter bounded below by $m_{G}[0,1)$ and above by $m_{G}[2, n]$. For example, one might ask if there exists a graph parameter $p(G)$ for which

$$
m_{G}[0,1) \leq p(G) \leq \gamma(G) .
$$

We considered three well-known graph parameters, each bounded above by $\gamma(G)$, and observed that they are not always bounded below by $m_{G}[0,1)$. More precisely, while the 2-packing number $\rho(G)$ (see [3]) is always at most $\gamma(G)$, we can find a graph for which $\rho(G)<m_{G}[0,1)$. Similar examples can be found for the fractional domination number $\gamma_{f}(G)$ [14], and the irredundance number $\operatorname{ir}(G)$ [11]. We omit the details.

One can also ask if there exists a graph parameter $q(G)$ for which

$$
\gamma(G) \leq q(G) \leq m_{G}[2, n]
$$

for isolate-free $G$. Graph parameters $q(G)$ for which $\gamma(G) \leq q(G)$ include the independent domination number $i(G)$, the edge covering number $\alpha_{1}(G)$, and the matching number $\beta_{1}(G)$. In the first two cases we can provide counter examples to show they are not necessarily bounded above by $m_{G}[2, n]$. Interestingly, we will see that $\gamma(G) \leq \beta_{1}(G) \leq m_{G}[2, n]$, when $G$ is isolate-free.

\section{UPPER BOUND FOR $\gamma(G)$}

In this section we show how to modify the proof of Theorem 1 to obtain a new inequality. For convenience, we recall the facts used to prove Theorem 1. Proofs or references can be found in [18]. In this paper, a star $S_{n}$ is the complete bipartite graph $K_{1, n-1}$, and $n \geq 2$.

Lemma 1. The star $S_{n}$ on $n$ vertices has Laplacian spectrum $0,1^{n-2}, n$. 
Lemma 2. For graphs $G_{1}=\left(V, E_{1}\right)$ and $G_{2}=\left(V, E_{2}\right)$ where $E_{1} \cap E_{2}=\emptyset$, and $G=\left(V, E_{1} \cup E_{2}\right)$, we have $L_{G}=L_{G_{1}}+L_{G_{2}}$.

Let $\lambda_{i}(A)$ denote the $i$-th largest eigenvalue of a Hermitian matrix $A$.

Lemma 3. If $A$ and $B$ are Hermitian matrices of order $n$, and $B$ is positive semidefinite, then $\lambda_{i}(A+B) \geq \lambda_{i}(A)$, for $1 \leq i \leq n$.

Lemma 4. Let $G=(V, E)$ and $H=(V, F)$ be graphs with $F \subseteq E$. Then

(1) for all $i, \mu_{i}(H) \leq \mu_{i}(G)$;

(2) for any $a, m_{H}[0, a) \geq m_{G}[0, a)$;

(3) for any $a, m_{H}[a, n] \leq m_{G}[a, n]$.

Let $S$ be a set of vertices, and $u \in S$. A vertex $v \in V-S$ is an external private neighbor of $u$ (with respect to $S$ ) if $N(v) \cap S=\{u\}$. That is, $v \in V-S$ is a neighbor of $u$, but not a neighbor of any other member of $S$.

Lemma 5 ([4]). Any graph without isolated vertices has a minimum dominating set in which every member has an external private neighbor.

We will say that $G$ has a star forest $F=\left(S_{n_{1}}, \ldots, S_{n_{k}}\right)$, if there exists a sequence of pairwise vertex-disjoint subgraphs $H_{i}$ of $G$, with $H_{i} \simeq S_{n_{i}}$, for all $i, 1 \leq i \leq k$. We emphasize that stars have order $n \geq 2$.

Lemma 6. Any isolate-free graph $G=(V, E)$ with domination number $\gamma$ has a star forest $F=\left(S_{n_{1}}, \ldots, S_{n_{\gamma}}\right)$ such that every $v \in V$ belongs to exactly one star, and the centers of the stars form a minimum dominating set.

Theorem 1 is a spectral lower bound for $\gamma(G)$. The key to its proof was to take the star forest that cover all vertices,

$$
F=\left(S_{n_{1}}, S_{n_{2}}, \ldots, S_{n_{\gamma(G)}}\right)
$$

guaranteed by Lemma 6. By Lemma $1 m_{S_{n_{i}}}[0,1)=1$, and so $m_{F}[0,1)=\gamma(G)$. By part (2) of Lemma 4 we have $\gamma(G)=m_{F}[0,1) \geq m_{G}[0,1)$.

If instead of counting the smallest eigenvalue in each star we count the largest, we can also obtain a spectral upper bound for $\gamma(G)$. Assume that $G$ is isolate-free. In the construction of $F$, each star $S_{k}$ contains $k \geq 2$ vertices. When $k=2$, the star has eigenvalues 0,2 . When $k \geq 3$, the star has eigenvalues $0,1^{k-2}, k$. So $m_{S_{n_{i}}}[2, n]=1$ for all $i$. Since these are disjoint stars, $m_{F}[2, n]=\gamma(G)$. By Lemma 4, part (3), $m_{F}[2, n] \leq m_{G}[2, n]$. We conclude that

Theorem 2. If $G$ is an isolate-free graph, then $\gamma(G) \leq m_{G}[2, n]$.

We will use some ideas from our proof of Theorem 2 to establish Theorem 10 and Theorem 11, later in Section 4. However, there is actually an alternative and simpler proof to Theorem 2 which we sketch. Recall that the matching number $\beta_{1}(G)$, is the size of a largest set of independent edges in $G$. We first claim that $\beta_{1}(G) \leq m_{G}[2, n]$ for any graph $G$. To see this, let $F$ be the subgraph of $G$ consisting of $\beta_{1}(G)$ disjoint $K_{2}$ 's and $n-2 \beta_{1}(G)$ isolated vertices. Then $m_{F}[2, n]=\beta_{1}(G)$. By part (3) of Lemma 4, we must have $\beta_{1}(G)=m_{F}[2, n] \leq m_{G}[2, n]$. Finally, it is known [17] that if $G$ is isolate-free then $\gamma(G) \leq \beta_{1}(G)$, and so Theorem 2 follows.

A connection between $\beta_{1}(G)$ and the number of Laplacian eigenvalues strictly greater than two was shown in 2001 by Ming and Wang [21. They proved that if $G$ is connected and $n>2 \beta_{1}(G)$, then $\beta_{1}(G) \leq m_{G}(2, n]$. 
Theorem 2 strengthens a recent result by Zhou, Zhou and Du [27] which says that for trees $T, m_{T}[0,2) \leq n-\gamma(T)$. Note that Theorem 2 requires $G$ be isolate-free while Theorem 1 does not. This happens because isolates in Theorem 1 can be disregarded as they increase both sides of the inequality by one. In Theorem 2 an isolate increases one side of the inequality but not the other. Theorem 1 and Theorem 2 imply

Corollary 1. If $G$ is isolate-free then $m_{G}[0,1) \leq \gamma(G) \leq m_{G}[2, n]$.

It seems interesting in its own right that

Corollary 2. If $G$ is isolate-free, then $m_{G}[0,1) \leq m_{G}[2, n]$.

When combined with a known lower bound on $m_{T}[0,2)$ for trees, Theorem 1 implies something interesting about the interval $[1,2)$.

Corollary 3. If $T$ is a tree, then $m_{T}[1,2) \geq\left\lceil\frac{n}{2}\right\rceil-\gamma(T)$.

Proof. We have

$$
\begin{aligned}
m_{T}[1,2) & =m_{T}[0,2)-m_{T}[0,1) \\
& \geq\left\lceil\frac{n}{2}\right\rceil-m_{T}[0,1) \\
& \geq\left\lceil\frac{n}{2}\right\rceil-\gamma(T)
\end{aligned}
$$

The first inequality follows by the bound $m_{T}[0,2) \geq\left\lceil\frac{n}{2}\right\rceil$ for trees given in [5, Thr. 4.1]. The second inequality follows from Theorem 1 .

\section{Applications}

Recall that the distance between vertices $u$ and $v$ is the number of edges in a shortest path between them, and the graph's diameter, $\operatorname{diam}(G)$, is the greatest distance between any two vertices. It is known [15] that for trees $T,\left\lfloor\frac{\operatorname{diam}(T)}{2}\right\rfloor$ is a lower bound for both $m_{T}(0,2)$ and $m_{T}(2, n]$. For $G$ connected, it is also known [17] that $\frac{1+\operatorname{diam}(G)}{3} \leq \gamma(G)$, so Theorem 2 implies

Corollary 4. For connected graphs $G, \frac{1+\operatorname{diam}(G)}{3} \leq m_{G}[2, n]$.

Nordhaus-Gaddum inequalities. A Nordhaus-Gaddum inequality is a bound on the sum or product of a parameter for a graph $G$ and its complement $\bar{G}$. For an overview of Nordhaus-Gaddum inequalities for domination-related parameters we refer to Chapter 10 in [17]. A result of Jaeger and Payan [19] says that if $G$ is a graph then

$$
\begin{aligned}
\gamma(G)+\gamma(\bar{G}) & \leq n+1 \\
\gamma(G) \gamma(\bar{G}) & \leq n
\end{aligned}
$$

and these bounds are tight. The following theorem by Cockayne and Hedetniemi characterizes when equality occurs in (1).

Theorem 3 ([10]). For any graph $G, \gamma(G)+\gamma(\bar{G}) \leq n+1$ with equality if and only if $G=K_{n}$ or $G=\bar{K}_{n}$.

We can use this to obtain the following:

Theorem 4. For any graph $G, m_{G}[0,1)+m_{\bar{G}}[0,1) \leq n+1$ with equality if and only if $G=K_{n}$ or $G=\bar{K}_{n}$. 
Proof. From Theorem 1 and (1) we must have

$$
m_{G}[0,1)+m_{\bar{G}}[0,1) \leq \gamma(G)+\gamma(\bar{G}) \leq n+1
$$

for any $G$. Since $m_{K_{n}}[0,1)=1$ and $m_{\bar{K}_{n}}[0,1)=n$, we must have equality if $G=K_{n}$ or $G=\bar{K}_{n}$. Conversely if $m_{G}[0,1)+m_{\bar{G}}[0,1)=n+1$, then $(3)$ forces $\gamma(G)+\gamma(\bar{G})=n+1$. By Theorem 3 it follows that $G=K_{n}$ or $G=\bar{K}_{n}$.

From Theorem 1 and (2) we also have

Theorem 5. For any graph $G, m_{G}[0,1) \cdot m_{\bar{G}}[0,1) \leq n$.

Recall [22, Theorem 3.6] that if $G$ has Laplacian eigenvalues

$$
0=\mu_{1} \leq \mu_{2} \leq \ldots \leq \mu_{n}
$$

then the Laplacian eigenvalues of $\bar{G}$ are:

$$
0, n-\mu_{n}, n-\mu_{n-1}, \ldots, n-\mu_{2}
$$

It follows that $m_{\bar{G}}[0,1)=m_{G}(n-1, n]+1$. Then from Theorem 5

$$
\begin{aligned}
m_{G}[0,1) \cdot m_{G}(n-1, n] & <m_{G}[0,1) \cdot\left(m_{G}(n-1, n]+1\right)= \\
m_{G}[0,1) \cdot m_{\bar{G}}[0,1) & \leq n .
\end{aligned}
$$

We have

Theorem 6. For any graph $G, m_{G}[0,1) \cdot m_{G}(n-1, n]<n$.

We conclude that any graph of order $n$ must have fewer than $\sqrt{n}$ Laplacian eigenvalues in at least one of the intervals $[0,1)$ or $(n-1, n]$.

Gallai-type theorems. A Gallai-type theorem has the form $x(G)+y(G)=n$ where $x(G)$ and $y(G)$ are graph parameters. There are exactly $n$ Laplacian eigenvalues, so the equation

$$
m_{G}[0,1)+m_{G}[1, n]=n
$$

can be regarded as a trivial Gallai-type theorem. A spanning forest of a graph $G$ is a spanning subgraph which contains no cycles. Let $\varepsilon(G)$ denote the maximum number of pendant edges in a spanning forest of $G$.

Theorem 7 ( Nieminen [24]). For any graph $G, \gamma(G)+\varepsilon(G)=n$.

Corollary 5. For any graph $G, \varepsilon(G) \leq m_{G}[1, n]$.

Proof. From Theorem 1 and (4) we know that

$$
n-\gamma(G) \leq m_{G}[1, n]
$$

the left side being $\varepsilon(G)$ by Theorem 7 .

Corollary 6. $\gamma(G)=m_{G}[0,1)$ if and only if $\varepsilon(G)=m_{G}[1, n]$.

Proof. This follows from (44) and Theorem 7

Berge [2] gives an early bound for $\gamma(G)$ :

$$
\gamma(G)+\Delta(G) \leq n
$$

where $\Delta$ denotes the maximum vertex degree. In [12] the authors study when equality in (6) occurs. Combining (5) and (6) give

Theorem 8. For any graph $G, m_{G}[1, n] \geq \Delta(G)$. 
As a simple application to Theorem 8 , suppose we are given a list $\sigma$

$$
0=\mu_{n} \leq \mu_{n-1} \leq \ldots \leq \mu_{1}
$$

of non-negative numbers and wish to know if there is a graph $G$ whose Laplacian spectrum is $\sigma$. Then Theorem 8 imposes a necessary condition on $G$. Let $B=\mid\{i$ : $\left.\mu_{i} \geq 1\right\} \mid$. Any graph $G$ such that $\operatorname{Spec}(G)=\sigma$ must have vertices whose degrees are bounded by $B$.

\section{Approximating $\gamma(G)$}

In this section we explain why it is hard to approximate $\gamma(G)$ with a polynomial computable spectral quantity of the form $m_{G}[a, b]$. We show that $m_{G}[0,1)$ and $m_{G}[2, n]$ do not even achieve logarithmic approximation ratios. Yet, for certain classes of graphs such as trees and $c$-cyclic graphs, $\frac{m_{G}[2, n]}{\gamma(G)}$ is bounded by a constant.

Inapproximability. It is well-known that the decision problem DOMINATING SET is NP-complete [13, even for planar graphs. In the approximation algorithm literature the problem is classified as class II in the taxonomy of NP-complete problems given in [1]. Roughly speaking, this means that approximating with better than a logarithmic ratio is hard. A problem is called quasi-NP-hard if a polynomial-time algorithm for it could be used to solve all NP problems in time $2^{\text {poly }(\log n)}$. Thus the notion is slightly weaker than NP-hard.

Lund and Yannakakis [20, Thr. 3.6] showed that it is quasi-NP-hard to compute a polynomial-time function $f(G) \geq \gamma(G)$ for which

$$
\frac{f(G)}{\gamma(G)} \leq c \log _{2} n
$$

when $0<c<\frac{1}{4}$. Letting $g(G)=\frac{f(G)}{c \log _{2} n}$, we see this is equivalent to computing a polynomial time $g(G) \leq \gamma(G)$ for which

$$
\frac{\gamma(G)}{g(G)} \leq c \log _{2} n .
$$

Good approximations of $\gamma(G)$ do exist. The fractional domination number $\gamma_{f}(G)$ can be computed in polynomial time using linear programming. Given a vertex ordering, we can compute in polynomial time an approximation $\gamma_{g}(G)$ for $\gamma(G)$ using the greedy domination algorithm. Clearly for any graph $G$,

$$
\gamma_{f}(G) \leq \gamma(G) \leq \gamma_{g}(G)
$$

In [8] Chappell, Gimbel and Hartman proved that $\frac{\gamma_{g}(G)}{\gamma_{f}(G)}$ is in $O(\log n)$. It follows that both $\frac{\gamma_{g}(G)}{\gamma(G)}$ and $\frac{\gamma(G)}{\gamma_{f}(G)}$ must also be in $O(\log n)$. Note this result does not contradict that of Lund and Yannakakis, provided the constants of proportionality are sufficiently large.

Example. We now construct an infinite sequence of graphs for which the ratio $\frac{\gamma(G)}{m_{G}[0,1)} \notin$ $O(\log n)$. Our construction uses the tree $T$ of order $n=65$, shown in Figure 1. It is known [18] that $m_{T}[0,1)=24$ and $\gamma(T)=25$.

Recall that the Cartesian product $G \times H$ of two graphs $G=(V, E)$ and $H=(W, F)$ is the graph with vertex set $V \times W$ for which $\left(v_{1}, w_{1}\right)$ and $\left(v_{2}, w_{2}\right)$ are adjacent if and only if $v_{1}=v_{2}$ and $w_{1} w_{2} \in F$ or $w_{1}=w_{2}$ and $v_{1} v_{2} \in E$. 


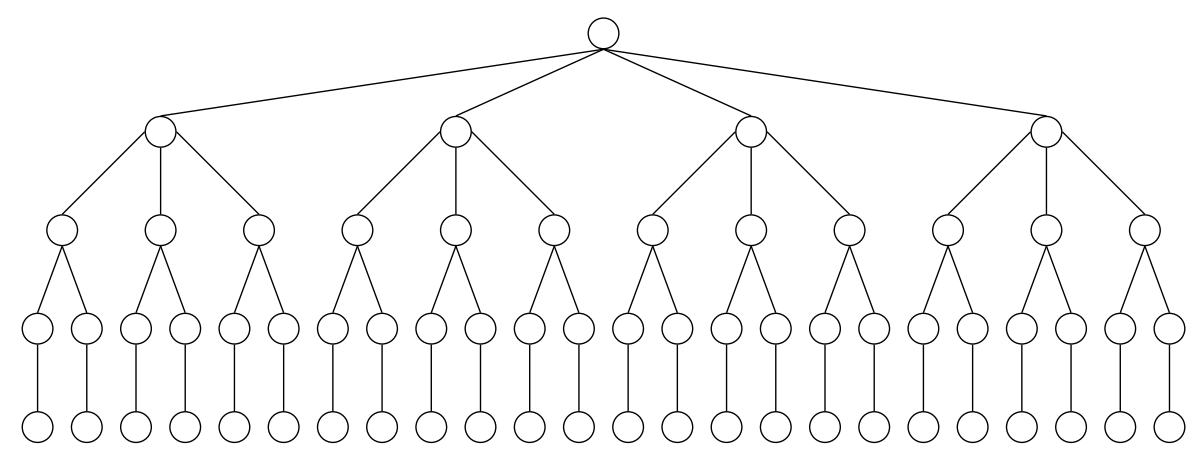

Figure $1 . \quad m_{T}[0,1)=24<\gamma(T)=25$

In 1968 V. G. Vizing conjectured [25] that for all graphs $G$ and $H$,

$$
\gamma(G) \cdot \gamma(H) \leq \gamma(G \times H)
$$

While this currently remains an open problem, many partial results exist. We say that $G$ satisfies Vizing's conjecture if (7) holds for all graphs $H$. Many classes of graphs are known to satisfy Vizing's conjecture.

Lemma 7 ( Theorem 8.2, [7). All trees satisfy Vizing's conjecture.

It is easy to show that the Cartesian product is an associative operation. Let $G^{k}$ denote the Cartesian product $G \times \ldots \times G$ of $k$ copies of $G$.

Lemma 8. If $G$ satisfies Vizing's conjecture, then $\gamma(G)^{k} \leq \gamma\left(G^{k}\right)$.

Proof. By induction on $k$, the case for $k=1$ being trivial. Assume that $\gamma(G)^{k} \leq \gamma\left(G^{k}\right)$. Using the induction assumption, the fact that $G$ satisfies Vizing's conjecture, and the associativity of $\times$, we have

$$
\gamma(G)^{k+1}=\gamma(G) \gamma(G)^{k} \leq \gamma(G) \gamma\left(G^{k}\right) \leq \gamma\left(G \times G^{k}\right)=\gamma\left(G^{k+1}\right)
$$

completing the proof.

The following is well-known (See, for example, [22, Thr. 3.5]).

Lemma 9. Let $G$ and $H$ be graphs with Laplacian spectra

$$
0=\mu_{n} \leq \mu_{n-1} \leq \ldots \leq \mu_{1}
$$

and

$$
0=\mu_{m}^{\prime} \leq \mu_{m-1}^{\prime} \leq \ldots \leq \mu_{1}^{\prime}
$$

respectively. Then the Laplacian spectrum of $G \times H$ is

$$
\left\{\mu_{i}+\mu_{j}^{\prime} \mid 1 \leq i \leq n, 1 \leq j \leq m\right\} .
$$

Lemma 10. For any graphs $G$ and $H, m_{G \times H}[0,1) \leq m_{G}[0,1) \cdot m_{H}[0,1)$.

Proof. By Lemma 9, Laplacian eigenvalues of $G \times H$ are of the form $\mu_{i}+\mu_{j}^{\prime}$, where $\mu_{i}$ and $\mu_{j}^{\prime}$ are eigenvalues of $G$ and $H$ respectively. A necessary condition for $\mu_{i}+\mu_{j}^{\prime}<1$ is that $\mu_{i}<1$ and $\mu_{j}^{\prime}<1$. There are at most $m_{G}[0,1) \cdot m_{H}[0,1)$ such pairs.

Lemma 11. For any graph $G$ and any $k \geq 1, m_{G^{k}}[0,1) \leq m_{G}[0,1)^{k}$. 
Proof. The case $k=1$ is trivial, and $k=2$ is handled by Lemma 10. Assume $m_{G^{k}}[0,1) \leq m_{G}[0,1)^{k}$. Then using Lemma 10 and the induction assumption, we have:

$$
m_{G^{k+1}}[0,1)=m_{G \times G^{k}}[0,1) \leq m_{G}[0,1) \cdot m_{G^{k}}[0,1) \leq m_{G}[0,1) \cdot m_{G}[0,1)^{k} .
$$

The right side is $m_{G}[0,1)^{k+1}$ completing the induction.

Let $T$ be the tree of order 65 in Figure 1 for which

$$
m_{T}[0,1)=24 \text { and } \gamma(T)=25 .
$$

We claim that for all $k \geq 1$

$$
m_{T^{k}}[0,1) \leq m_{T}[0,1)^{k} \leq \gamma(T)^{k} \leq \gamma\left(T^{k}\right)
$$

The first inequality follows by Lemma 11, and the second inequality follows by Theorem 1. The third inequality follows by Lemma 7 and Lemma 8 ,

Theorem 9. There exists a sequence of graphs $G_{k}$ with $\frac{\gamma\left(G_{k}\right)}{m_{G_{k}}[0,1)} \notin O(\log n)$.

Proof. We let $G_{k}=T^{k}$. Using $n=65^{k}$, (8) and (9) we have

$$
\frac{\gamma\left(T^{k}\right)}{m_{T^{k}}[0,1)} \geq \frac{\gamma(T)^{k}}{m_{T}[0,1)^{k}}=\left(\frac{25}{24}\right)^{k}=\left(\frac{25}{24}\right)^{\log _{65} n}=n^{\log _{65} \frac{25}{24}}=n^{.009779} .
$$

Ratios for certain classes. Consider the two approximation ratios:

$$
\begin{aligned}
& \frac{\gamma(G)}{m_{G}[0,1)} \\
& \frac{m_{G}[2, n]}{\gamma(G)}
\end{aligned}
$$

Both ratios can get arbitrarily large. By Theorem 9 the first of these ratios is not bounded by $\log (n)$. The second ratio also gets arbitrarily large. When $G=K_{n}$ is the complete graph, we see that ratio (11) is $n-1$.

Consider (11) for paths $P_{n}$. It is well-known that $\gamma\left(P_{n}\right)=\left\lceil\frac{n}{3}\right\rceil$. By Thr. 4.1 in [5] we also know $m_{P_{n}}[2, n] \leq\left\lfloor\frac{n}{2}\right\rfloor$, and so (11) is at most $\frac{3}{2}$. Using ideas from Section 2, we show that for all trees ratio (11) is less than two.

Lemma 12. Let $G$ be a graph on $n$ vertices and $m<\left(\begin{array}{l}n \\ 2\end{array}\right)$ edges, and let $G^{\prime}$ be the graph obtained by adding an edge. Then for any $a \geq 0$,

$$
m_{G}[a, n] \leq m_{G^{\prime}}[a, n] \leq m_{G}[a, n]+1 .
$$

Proof. Let $0=\mu_{n} \leq \ldots \leq \mu_{2} \leq \mu_{1}$ and $0=\mu_{n}^{\prime} \leq \ldots \leq \mu_{2}^{\prime} \leq \mu_{1}^{\prime}$ be the respective Laplacian spectra of $G$ and $G^{\prime}$. By the well-know interlacing theorem [16, Thr. 2.4] for Laplacian eigenvalues we know

$$
0=\mu_{n}=\mu_{n}^{\prime} \leq \ldots \leq \mu_{k} \leq \mu_{k}^{\prime} \leq \ldots \leq \mu_{2} \leq \mu_{2}^{\prime} \leq \mu_{1} \leq \mu_{1}^{\prime}
$$

If $a=0$, then $m_{G}[a, n]=m_{G^{\prime}}[a, n]=n$. If $\mu_{1}<a$ then $m_{G}[a, n]=m_{G^{\prime}}[a, n]=0$. We may assume that $0<a \leq \mu_{1}$. Choose $k$ to be the largest index for which $a \leq \mu_{k}$. Then $\mu_{k+1}<a \leq \mu_{k}$. There is a single eigenvalue of $G^{\prime}$, namely $\mu_{k+1}^{\prime}$ in $\left[\mu_{k+1}, \mu_{k}\right]$. If $\mu_{k+1}^{\prime} \leq a$, then $m_{G^{\prime}}[a, n]=m_{G}[a, n]+1$. Otherwise, $m_{G^{\prime}}[a, n]=m_{G}[a, n]$.

Theorem 10. If $T$ is a tree, then $1 \leq \frac{m_{T}[2, n]}{\gamma(T)}<2$. 
Proof. Let $F=\left(S_{n_{1}}, \ldots, S_{n_{\gamma}}\right)$ be the star forest guaranteed by Lemma 6. Then $m_{F}[2, n]$ is exactly $\gamma(T)$. Starting with $F$, we can construct $T$ by adding $\gamma(T)-1$ edges. By Lemma 12 the addition of each edge can increase $m_{T}[2, n]$ by at most one. Therefore

$$
m_{F}[2, n] \leq m_{T}[2, n] \leq m_{F}[2, n]+\gamma(T)-1 .
$$

But the right side is $2 \gamma(T)-1$ and the theorem follows.

A connected graph having $n-1+c$ edges is called $c$-cyclic. We can generalize Theorem 10 as follows.

Theorem 11. If $G$ is c-cyclic, $c \geq 1$, then $1 \leq \frac{m_{G}[2, n]}{\gamma(G)} \leq c+1$.

Proof. Let $F=\left(S_{n_{1}}, \ldots, S_{n_{\gamma}}\right)$ be the star forest in $G$ from Lemma 6. Then we may select $\gamma(G)-1$ additional edges to form a spanning tree $T$. Since $T$ has $n-1$ edges, there must be $c$ remaining edges. Therefore $G$ can be constructed from $F$ by adding $\gamma(G)-1+c$ edges. By Lemma 12

$$
m_{G}[2, n] \leq m_{F}[2, n]+\gamma(G)-1+c=2 \gamma(G)+c-1,
$$

or

$$
\frac{m_{G}[2, n]}{\gamma(G)} \leq 2+\frac{c-1}{\gamma(G)} \leq 2+c-1,
$$

the last inequality holding because $c \geq 1$ and $\gamma(G) \geq 1$.

Let us now consider ratio (10) for trees. For the tree in Figure 1, the ratio (10) is $\frac{25}{24}$. It is possible to generalize this example. We construct the tree $T_{k}$ on $65 k+1$ vertices by taking $k$ copies of this tree, and adjoining the root to each copy. Using the algorithm in [5], it is straightforward to determine that $m_{T_{k}}[0,1)=24 k$. Using the domination algorithm in [9] it can be shown that $\gamma\left(T_{k}\right)=25 k$. Thus, the difference between $\gamma\left(T_{k}\right)-m_{T_{k}}[0,1)$ grows arbitrarily large. However, the ratio (10) remains at $\frac{25}{24}$. In all known examples of trees ratio (10) is either 1 or $\frac{25}{24}$, and it is tempting to conjecture that the ratio is bounded by a constant for trees.

\section{CONCLUding REMARKS}

Many of the results of this paper also apply to the signless Laplacian spectrum. For example, if we let $m_{G}^{+} I$ denote the number of signless Laplacian eigenvalues of $G$ in $I$, then Theorem 1 and Theorem 2 are also true if we replace $m_{G}$ with $m_{G}^{+}$.

We conclude by suggesting two problems for further study. First, characterize those graphs $G$ for which $m_{G}[0,1)=\gamma(G)$. Second, determine if $\frac{\gamma(T)}{m_{T}[0,1)}$ bounded by a constant for trees $T$.

\section{REFERENCES}

1. Sanjeev Arora and Carsten Lund, Hardness of approximations, Approximation Algorithms for NPHard Problems (Dorit S. Hochbaum, ed.), PWS Publishing Company, Boston, 1997, pp. 399-446.

2. Claude Berge, Graphs and hypergraphs, North-Holland Publishing Co., Amsterdam-London; American Elsevier Publishing Co., Inc., New York, 1973, Translated from the French by Edward Minieka, North-Holland Mathematical Library, Vol. 6.

3. Thomas Böhme and Bojan Mohar, Domination, packing and excluded minors, Electron. J. Combin. 10 (2003), Note 9, 6 pp. (electronic).

4. Béla Bollobás and Ernie J. Cockayne, Graph-theoretic parameters concerning domination, independence, and irredundance, J. Graph Theory 3 (1979), no. 3, 241-249.

5. Rodrigo O. Braga, Virgínia M. Rodrigues, and Vilmar Trevisan, On the distribution of Laplacian eigenvalues of trees, Discrete Math. 313 (2013), no. 21, 2382-2389. 
6. Clemens Brand and Norbert Seifter, Eigenvalues and domination in graphs, Math. Slovaca 46 (1996), no. 1, 33-39.

7. Boštjan Brešar, Paul Dorbec, Wayne Goddard, Bert L. Hartnell, Michael A. Henning, Sandi Klavžar, and Douglas F. Rall, Vizing's conjecture: a survey and recent results, J. Graph Theory 69 (2012), no. 1, 46-76.

8. Glenn G. Chappell, John Gimbel, and Chris Hartman, Approximations of the domination number of a graph, preprint, 2005.

9. Ernie J. Cockayne, S. Goodman, and Stephen T. Hedetniemi, A linear time algorithm for the domination number of a tree, Inf. Proc. Lett. 4 (1975), no. 2, 41-44.

10. Ernie J. Cockayne and Stephen T. Hedetniemi, Toward a theory of domination in graphs, Networks 7 (1977), 247-261.

11. Peter Damaschke, Irredundance number versus domination number, Discrete Math. 89 (1991), no. $1,101-104$.

12. Gayla S. Domke, Jean E. Dunbar, and Lisa R. Markus, Gallai-type theorems and domination parameters, Discrete Math. 167/168 (1997), 237-248, 15th British Combinatorial Conference (Stirling, 1995).

13. Michael R. Garey and David S. Johnson, Computers and intractability, W. H. Freeman and Co., San Francisco, Calif., 1979, A guide to the theory of NP-completeness, A Series of Books in the Mathematical Sciences.

14. Dana L. Grinstead and Peter J. Slater, Fractional domination and fractional packing in graphs, Proceedings of the Twentieth Southeastern Conference on Combinatorics, Graph Theory, and Computing (Boca Raton, FL, 1989), vol. 71, 1990, pp. 153-172.

15. Robert Grone, Russell Merris, and V. S. Sunder, The Laplacian spectrum of a graph, SIAM J. Matrix Anal. Appl. 11 (1990), no. 2, 218-238.

16. Frank J. Hall, Kinnari Patel, and Michael Stewart, Interlacing results on matrices associated with graphs, J. Combin. Math. Combin. Comput. 68 (2009), 113-127.

17. Teresa W. Haynes, Stephen T. Hedetniemi, and Peter J. Slater, Fundamentals of domination in graphs, Monographs and Textbooks in Pure and Applied Mathematics, vol. 208, Marcel Dekker, Inc., New York, 1998.

18. Stephen T. Hedetniemi, David P. Jacobs, and Vilmar Trevisan, Domination number and Laplacian eigenvalue distribution, European J. Combin. 53 (2016), 66-71.

19. François Jaeger and Charles Payan, Relations du type Nordhaus-Gaddum pour le nombre d'absorption d'un graphe simple, C. R. Acad. Sci. Paris Sér. A-B 274 (1972), A728-A730.

20. Carsten Lund and Mihalis Yannakakis, On the hardness of approximating minimization problems, J. Assoc. Comput. Mach. 41 (1994), no. 5, 960-981.

21. Guo Ji Ming and Tan Shang Wang, A relation between the matching number and Laplacian spectrum of a graph, Linear Algebra Appl. 325 (2001), no. 1-3, 71-74.

22. Bojan Mohar, The Laplacian spectrum of graphs, Graph theory, combinatorics, and applications. Vol. 2 (Kalamazoo, MI, 1988), Wiley-Intersci. Publ., Wiley, New York, 1991, pp. 871-898.

23. — Laplace eigenvalues of graphs - a survey, Discrete Math. 109 (1992), no. 1-3, 171-183, Algebraic graph theory (Leibnitz, 1989).

24. J. Nieminen, Two bounds for the domination number of a graph, J. Inst. Math. Appl. 14 (1974), $183-187$.

25. Vadim G. Vizing, Some unsolved problems in graph theory, Uspehi Mat. Nauk 23 (1968), no. 6 (144), 117-134.

26. Rundan Xing and Bo Zhou, Laplacian and signless Laplacian spectral radii of graphs with fixed domination number, Math. Nachr. 288 (2015), no. 4, 476-480.

27. Lingling Zhou, Bo Zhou, and Zhibin Du, On the number of Laplacian eigenvalues of trees smaller than two, Taiwanese J. Math. 19 (2015), no. 1, 65-75. 
Departamento de Matemática, Univ. De Aveiro, 3810-193 Aveiro, Portugal

E-mail address: dcardoso@ua.pt

School of Computing, Clemson University Clemson, SC 29634 USA

E-mail address: dpj@clemson.edu

Instituto de Matemática, UfRGS, 91509-900 Porto Alegre, RS, Brazil

E-mail address: trevisan@mat.ufrgs.br 